\title{
Estudo dos achados oculares na síndrome de Stevens-Johnson em pacientes de centro de referência de atendimento terciário
}

\author{
Study of the ocular findings in Stevens-Johnson syndrome patients \\ from a tertiary ophthalmologic center
}

\author{
Marciel Dourado Franca ${ }^{1}$ \\ Jane Palma Galrão Lima ${ }^{2}$ \\ Denise de Freitas ${ }^{3}$ \\ MarceloCunha ${ }^{4}$ \\ José Álvaro Pereira Gomes ${ }^{5}$
}

Trabalho realizado no Departamento de Oftalmologia da Universidade Federal de São Paulo - UNIFESP - São Paulo (SP) - Brasil.

${ }^{1}$ Médico Oftalmologista.

${ }^{2}$ Médica Oftalmologista.

${ }^{3}$ Livre Docente e Doutor em Oftalmologia pelo Departamento de Oftalmologia da Universidade Federal de São Paulo - UNIFESP - São Paulo (SP) - Brasil.

${ }^{4}$ Doutor em Oftalmologia. Médico assistente do Departamento de Oftalmologia da UNIFESP - São Paulo (SP) - Brasil.

${ }^{5}$ Professor Livre Docente do Setor de Córnea e Doenças Externas do Departamento de Oftalmologia da UNIFESP - São Paulo (SP) - Brasil.

Endereço para correspondência: Marciel Dourado Franca. Praça Mário Dourado, 198 - Irecê (BA) CEP 44900-000

E-mail: marcielfranca@uol.com.br

Recebido para publicação em 19.06.2005

Última versão recebida em 12.03.2009

Aprovação em 11.05.2009

Nota Editorial: Depois de concluída a análise do artigo sob sigilo editorial e com a anuência do Dr. Renato Ambrósio Júnior sobre a divulgação de seu nome como revisor, agradecemos sua participação neste processo.

\begin{tabular}{|c|}
\hline RESUMO \\
\hline 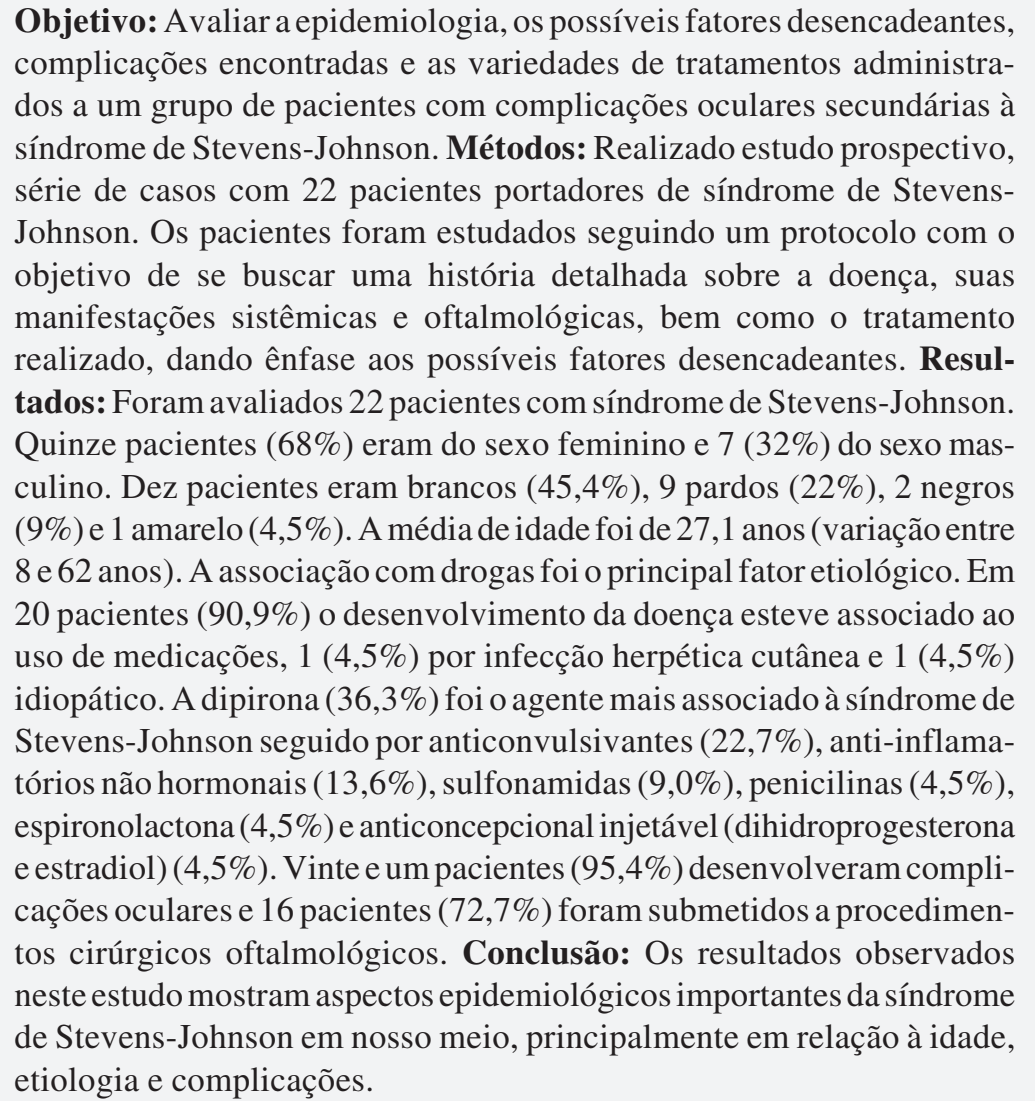 \\
\hline
\end{tabular}

Descritores: Síndrome de Stevens-Johnson/epidemiologia; Hipersensibilidade a drogas Opacidade da córnea, Acuidade visual

\section{INTRODUÇÃO}

A síndrome de Stevens-Johnson (SSJ) é definida como uma doença inflamatória aguda, febril e autolimitada, com duração aproximada de duas a quatro semanas que afeta a pele e membranas mucosas. $\mathrm{O}$ envolvimento cutâneo é caracterizado por lesões arredondadas em forma de alvo as quais medem menos que 3 milímetros de diâmetro e que geralmente comprometem menos que $20 \%$ da área corporal ${ }^{(1)}$. A erupção bolhosa inflamatória pode 
envolver a mucosa orofaríngea, pálpebras, conjuntivas, genitais e vísceras ${ }^{(1-3)}$.

A doença ocular aguda é caracterizada por uma conjuntivite purulenta catarral bilateral, membranosa ou pseudomembranosa que frequentemente está associada à ulceração corneana e uveíte anterior ${ }^{(1)}$. Durante a fase crônica, a maioria dos pacientes apresenta numerosas alterações da superfície ocular, destacando: simbléfaro, entrópio, ectrópio, triquíase, olho seco, inflamação conjuntival persistente, conjuntivalização corneana e queratinização ${ }^{(4)}$.

A síndrome inicia-se, geralmente, após o uso de medicações ou ocorrência de infecções e provavelmente apresenta etiopatogenia autoimune. A literatura relata mais de 100 drogas associadas com a etiologia desta afecção, sendo as mais comumente relacionadas: sulfonamidas, anticonvulsivantes, anti-inflamatórios não-hormonais e alopurinol ${ }^{(2-3,5-6)}$. Dentre os agentes infecciosos, destacam-se Mycoplasma pneumoniae, herpes simples e adenovirus ${ }^{(3,5,7)}$.

A incidência da SSJ está estimada entre 1 a 6 casos por milhão de habitantes ${ }^{(5)}$. Apesar de rara, esta doença gera um forte impacto econômico e social, uma vez que se trata de uma entidade crônica que leva potencialmente à cegueira pacientes numa faixa etária muitas vezes produtiva.

O objetivo primário deste estudo é descrever os possíveis fatores desencadeantes implicados, bem como as complicações oftalmológicas e as variedades de tratamento administrados a um grupo de pacientes com envolvimento ocular secundário à síndrome de Stevens-Johnson.

\section{MÉTODOS}

No período compreendido entre janeiro a dezembro de 2000, 22 pacientes consecutivos, portadores de SSJ (fase crônica), iniciaram acompanhamento no setor de Doenças Externas Oculares e Córnea do Departamento de Oftalmologia da Escola Paulista de Medicina - Universidade Federal de São Paulo.

Como critério de inclusão, os pacientes deveriam apresentar uma história prévia de enfermidade mucocutânea com as características de lesões em forma de alvo, bolhas e extensas áreas de necrose, com uma fase prodrômica aguda proeminente e envolvimento de no mínimo dois sítios de mucosa. Todos os pacientes foram estudados seguindo um protocolo e questionário com o objetivo de se buscar uma história detalhada sobre a doença, suas manifestações sistêmicas e oftalmológicas, bem como o tratamento realizado, dando ênfase aos possíveis fatores desencadeantes. O protocolo incluiu dados demográficos, antecedentes pessoais e familiares, história prévia de uso de drogas, tempo de internação durante a fase aguda, atendimento oftalmológico na crise aguda, uso de corticosteróides tópico e sistêmico, manifestações sistêmicas associadas, envolvimento cutâneo e de mucosas, exame oftalmológico completo (quantificação da acuidade visual, biomicroscopia, pressão intraocular, fundo de olho, teste de
Schirmer sem anestésico) e tipo de procedimento cirúrgico realizado. Todos os dados descritivos do questionário foram obtidos a partir das informações dadas pelos pacientes ou por familiares.

\section{RESULTADOS}

Neste estudo, foram identificados 22 pacientes com síndrome de Stevens-Johnson, dos quais 21 desenvolveram complicações oculares. Quinze pacientes (68\%) eram do sexo feminino e $7(32 \%)$ do sexo masculino. Dez pacientes eram brancos $(45,4 \%), 1$ amarelo (4,5\%), 2 negros (9\%) e 9 pardos (22\%). A média de idade foi de 27,1 anos (variação entre 8 e 62 anos).

A associação com drogas foi o principal fator desencadeante no surgimento da SSJ. Do total de 22 pacientes, 20 $(90,9 \%)$ estavam associados ao uso de medicações, $1(4,5 \%)$ por infecção herpética cutânea e $1(4,5 \%)$ idiopático. A dipirona $(36,3 \%)$ foi o agente mais comumente associado à SSJ, seguido em ordem decrescente pelos anticonvulsivantes $(22,7 \%)$, anti-inflamatórios não-hormonais (AINH) $(13,6 \%)$, sulfonamidas $(9,0 \%)$, penicilinas $(4,5 \%)$, espironolactona $(4,5 \%)$ e anticoncepcional injetável (dihidroprogesterona e estradiol) $(4,5 \%)$ (Tabela 1$)$.

Dos 22 pacientes com envolvimento ocular a cavidade oral foi comprometida em $100 \%$ dos casos, seguido pelos órgãos genitais em $15(68,1 \%)$. Vinte e um pacientes desenvolveram alguma complicação ocular, como entrópio, triquíase, simbléfaro, anquilobléfaro, metaplasia epitelial conjuntival, leucoma e neovascularização corneana. Um paciente apresentou a típica conjuntivite catarral membranosa, mas não apresentou sequelas na fase crônica.

Dezesseis pacientes $(72,7 \%)$ foram submetidos a algum tipo de procedimento cirúrgico com a finalidade de melhorar e/ou restaurar a superfície externa ocular. (Tabela 2).

Todos os pacientes ficaram internados durante a fase aguda com o período médio de internação de 26,3 dias (variação entre 0 e 60 dias). Nesta ocasião, 5 pacientes $(22,7 \%)$ receberam corticosteróide tópico ocular, $1(4,5 \%)$ recebeu corticosteróide tópico e sistêmico e $4(18,2 \%)$ receberam só corticosteróide sistêmico. Treze pacientes (59\%) não sabiam informar a medicação usada durante a internação hospitalar.

A média do teste de Schirmer I no olho direito foi de 10,95 mm (variação de 0 a $30 \mathrm{~mm}$ ) e no olho esquerdo de 9,61 (variação de 0 a $30 \mathrm{~mm}$ ).

Oito pacientes $(36,3 \%)$ foram atendidos por oftalmologistas durante a fase aguda. A acuidade visual variou em ambos os olhos de sem percepção e projeção de luz (SPL) a 1,0. Os dados inerentes à acuidade visual estão listados na tabela 1 .

\section{DISCUSS ÃO}

Ferdinand von Hebra*, em 1866, descreveu uma síndrome cutânea auto-limitada, recorrente, caracterizada por lesões cu- 


\begin{tabular}{|c|c|c|c|c|c|c|c|c|}
\hline $\mathbf{P}$ & I & $\mathbf{s}$ & Fator desencadeante & M.A. & S.I OD/OE & E.O.F.A. & CT/CS & AV OD/OE \\
\hline 1 & 10 & $\mathrm{~F}$ & Espironolactona & $\mathrm{C} / \mathrm{O}$ & $10 / 13$ & Não & Não/Sim & $0,50 / 0,50$ \\
\hline 2 & 46 & M & Dipirona & $\mathrm{C} / \mathrm{O}$ & 07/02 & Não & Não/Sim & 0,40/PPL \\
\hline 3 & 10 & $\mathrm{~F}$ & Fenitoína & $\mathrm{T}$ & $09 / *$ & Não & $?$ & $0,50 / *$ \\
\hline 4 & 48 & M & Sulfonamida & $\mathrm{T}$ & $05 / 24$ & Não & $?$ & $\mathrm{CD} / 0,67$ \\
\hline 5 & 32 & $\mathrm{~F}$ & Dipirona & $\mathrm{C} / \mathrm{O}$ & $10 / 30$ & Sim & $?$ & MM/CD \\
\hline 6 & 18 & $\mathrm{~F}$ & Carbamazepina & $\mathrm{C} / \mathrm{O}$ & $07 / 03$ & Não & $?$ & $0,33 / 0,25$ \\
\hline 7 & 26 & $\mathrm{~F}$ & D. e Estradiol & $\mathrm{T}$ & $02 / 00$ & Não & $?$ & $0,10 / 0,10$ \\
\hline 8 & 17 & $\mathrm{~F}$ & Dipirona & $\mathrm{T}$ & $00 / 03$ & Não & $?$ & PPL/0,05 \\
\hline 9 & 18 & $\mathrm{~F}$ & Dipirona & $\mathrm{T}$ & $22 / 12$ & Não & $?$ & $\mathrm{MM} / 1,00$ \\
\hline 10 & 25 & $\mathrm{~F}$ & Penicilina & $\mathrm{T}$ & $10 / 18$ & Não & Não/Sim & $C D / 1,00$ \\
\hline 11 & 8 & $M$ & Herpes simples & $\mathrm{T}$ & $20 / 10$ & Não & $?$ & $0,67 / 0,10$ \\
\hline 12 & 34 & $\mathrm{~F}$ & Dipirona & $\mathrm{T}$ & $20 / 00$ & Sim & $?$ & $0,20 / 0,20$ \\
\hline 13 & 26 & $\mathrm{~F}$ & Fenitoína & $\mathrm{T}$ & $22 / 09$ & Não & $?$ & $1,00 / 0,33$ \\
\hline 14 & 58 & $M$ & Indeterminado & $\mathrm{T}$ & $05 / 00$ & Sim & $?$ & $0,20 / C D$ \\
\hline 15 & 28 & $\mathrm{~F}$ & Dipirona & $\mathrm{T}$ & $07 / 10$ & Sim & Sim/Não & PPL/0,25 \\
\hline 16 & 8 & $M$ & Dipirona & $\mathrm{T}$ & $30 / 20$ & Não & $?$ & $0,05 / 0,05$ \\
\hline 17 & 10 & $\mathrm{~F}$ & Fenobarbital & $\mathrm{C} / \mathrm{O}$ & $16 / 17$ & Sim & Sim/Não & $0,67 / 0,67$ \\
\hline 18 & 30 & $\mathrm{~F}$ & Diclofenaco & $\mathrm{C} / \mathrm{O}$ & 06/04 & Sim & Sim/Não & $0,05 / 0,05$ \\
\hline 19 & 26 & M & Dipirona & $\mathrm{T}$ & $20 / 18$ & Não & $?$ & PPL/PPL \\
\hline 20 & 62 & $M$ & Diclofenaco & $\mathrm{C} / \mathrm{O}$ & $00 / 07$ & Sim & Sim/Sim & SPL/MM \\
\hline 21 & 13 & $\mathrm{~F}$ & Sulfonamida & $\mathrm{T}$ & 05/02 & Sim & Não/Não & $0,40 / C D$ \\
\hline 22 & 45 & $\mathrm{~F}$ & Diclofenaco & $\mathrm{T}$ & $08 / 00$ & Não & Sim/Não & PPL/PPL \\
\hline \multicolumn{9}{|c|}{$\begin{array}{l}\mathrm{P}=\text { pacientes; } \mathrm{I}=\text { idade; } \mathrm{S}=\text { sexo; } \mathrm{M}=\text { masculino; } \mathrm{F}=\text { feminino; } \mathrm{M} . \mathrm{A}=\text { mucosas acometidas; } \mathrm{S} . \mathrm{I}=\text { teste de } \mathrm{Schirmer} \mathrm{I} ; \mathrm{OD}=\mathrm{olho} \text { direito; } \mathrm{OE}=\text { olho esquerdo; } \mathrm{E} . \mathrm{O} . \mathrm{F} . \mathrm{A}=\text { exame } \\
\text { oftalmológico na fase aguda; } \mathrm{CT}=\text { corticosteróide tópico; } \mathrm{CS}=\text { corticosteróide sistêmico; } \mathrm{AV}=\text { acuidade visual; } \mathrm{C}=\text { conjuntival; } \mathrm{O}=\mathrm{Oral} ; \mathrm{T}=\text { comprometimento de todas } \\
\text { as mucosas; } \mathrm{PPL}=\text { percepção e projeção luminosa; }=\text { olho eviscerado; } \mathrm{CD}=\text { conta dedos; } \mathrm{MM}=\text { movimentos de mãos; ?= não sabiam informara medicação usada previamente; } \\
\mathrm{D} \text { = = dihidroprogesterona; } \mathrm{SPL}=\text { sem percepção luminosa }\end{array}$} \\
\hline
\end{tabular}

tâneas eritematosas arredondadas. Em 1922, Stevens e Johnson" descreveram "uma nova febre eruptiva com estomatite e oftalmia", que representa um espectro mais grave do eritema multiforme de von Hebra. Em 1950, Thomas** sugeriu a divisão em duas formas: eritema multiforme minor (von Hebra) e eritema multiforme major (Stevens-Johnson). Em 1956, Lyell ${ }^{* *}$ descreveu uma condição clínica caracterizada pela perda epidérmica extensiva em paciente com síndrome de StevensJohnson, a qual ele chamou de necrólise epidérmica tóxica ${ }^{(1-2)}$.

A proposta deste estudo foi avaliar a epidemiologia, os possíveis fatores desencadeantes, complicações encontradas e as variedades de tratamentos administrados ao grupo de pacientes com complicações oculares secundárias à síndrome de Stevens-Johnson.

Dos 22 pacientes estudados, todos referiram terem apresentado manifestações oculares durante a fase aguda - período que compreende, aproximadamente, o tempo de internação hospitalar. Vinte e um pacientes desenvolveram complicações oculares na fase crônica da doença.

A fase aguda da SSJ normalmente dura de 2 a 6 semanas. Inicialmente, as pálpebras apresentam-se edemaciadas e eritematosas, a conjuntiva apresenta hiperemia difusa e bolhas subepiteliais podem ocorrer, embora estas raramente possam ser observadas. Após o estágio vesicular agudo, inicia-se um

\footnotetext{
* von Hebra (1866); Stevens e Johnson (1922) apud ${ }^{(1)}$

** Thomas (1950); Lyell (1956) apud ${ }^{(2)}$
}

quadro de conjuntivite catarral purulenta e sua gravidade é usualmente paralela à erupção cutânea.

O envolvimento mais grave é representado por uma conjuntivite membranosa ou pseudomembranosa. A cicatrização

\begin{tabular}{|clc|}
\hline \multicolumn{3}{|c|}{ Tabela 2. Procedimentos cirúrgicos realizados } \\
Pac. & Tipos de & Tempo \\
& procedimentos & CA X TP \\
2 & Txml / Patch, Txma, tarsorrafia / Txl, Txma & 4 anos \\
5 & Epilação de cílios & 4 anos \\
7 & Rtima & - \\
8 & Cirurgia de reconstrução da superfície ocular & - \\
9 & Adesivo tecidual, Tx tectônico & 3 meses \\
10 & Epilação de cílios & 5 anos \\
11 & Patch de córnea & 4 meses \\
12 & Adesivo tecidual & 19 anos \\
13 & Cirurgia de reconstrução da superfície ocular & 2 anos \\
14 & Epilação de cílios & 13 anos \\
15 & Tarsorrafia & 13 anos \\
16 & Epilação de cílios & 2 anos \\
19 & Transplante tríplice, Txl, Txma & 21 anos \\
20 & Reconstrução palpebral, Txml, tarsorrafia & - \\
21 & Rotação marginal, liberação de simbléfaro & - \\
22 & Txma & - \\
Pac.= paciente; Tx= transplante; Txml= transplante de mucosa labial; Txma= \\
transplante de membrana amniótica; Txl= transplante de limbo; Rtima= \\
recobrimento do tarso inferior commembrana amniótica; CAXTP=crise aguda \\
versus tempo do primeiro procedimento cirúrgico; -= dado não estimado \\
\hline
\end{tabular}


tecidual pode levar ao surgimento de simbléfaro ou anquilobléfaro e tardiamente, a entrópio, triquíase, conjuntivalização e/ou ceratinização corneana, lagoftalmo e olho $\operatorname{seco}^{(3,8-10)}$.

Dentre as complicações oculares observadas na fase crônica, neovascularização da córnea foi a mais comum, seguida por leucoma, simbléfaro, conjuntivalização corneana, entrópio, triquíase e anquilobléfaro. Apenas um paciente não apresentou uma destas sequelas na fase crônica. Provavelmente, estes dados refletem o fato do nosso serviço compreender um centro de referência terciário no atendimento de doenças oculares.

Drogas medicamentosas são os fatores precipitantes mais frequentes ${ }^{(5)}$. Os antibióticos são os agentes desencadeantes com maiores implicações e dentre estes as sulfonamidas são os que mais comumentes estão implicados seguido pelos anticonvulsivantes e $\mathrm{AINH}^{(5)}$. No nosso estudo observamos que, dentre os fatores desencadeantes, a associação da SSJ com o uso de medicações foi a mais comum. A literatura cita como drogas mais relacionadas: sulfonamidas, penicilinas, barbitúricos e anticonvulsivantes ${ }^{(3,5-6)}$. Entretanto, contrário a relatos da literatura, observamos que a dipirona foi responsável por (36,3\%) dos casos, seguidos pelos anticonvulsivantes $(22,7 \%)$, AINH $(13,6 \%)$, sulfonamidas $(9,0 \%)$, penicilinas $(4,5 \%)$ herpes simples $(4,5 \%)$, espironolactona $(4,5 \%)$ e dihiroprogesterona e estradiol (4,5\%). Não há dado semelhante descrito e a nossa alta incidência da dipirona como fator precipitante deve-se provavelmente ao fato deste analgésico ser rotineiramente utilizado no Brasil, fato que não ocorre por exemplo, nos EUA, onde esta droga tem o seu uso proibido pelo "Food and Drug Administration" (FDA). Este dado também pode representar um erro de interpretação, pois a dipirona é um medicamento utilizado de rotina em quadros febris, como na fase aguda da SSJ.

Recorrências de SSJ podem ocorrer se houver uma nova exposição ao agente causal. Em muitos casos, estão associados à infecção cutânea pelo vírus herpes simples.

Alguns autores relataram um grupo de pacientes que desenvolveram episódios recorrentes de inflamação conjuntival não associado a estímulos externos ${ }^{(11)}$. No presente estudo, observamos a recorrência da inflamação em dois casos. Um caso provavelmente secundário à infecção herpética e outro após cirurgia esofágica de causa indeterminada.

Não há tratamento específico para SSJ, entretanto deve se ter atenção especial às medidas de suporte e à orientação do paciente e familiares sobre a necessidade de o paciente evitar novamente o uso da medicação que causou a afecção.

É consenso que o oftalmologista deva acompanhar o paciente na fase aguda, realizando lise das membranas diariamente e examinando o paciente frequentemente, pois não é raro que os casos mais graves desenvolvam infecção ou perfuração corneana ${ }^{(8,12)}$. Outra importante modalidade de tratamento é o uso de lubrificantes, de preferência sem preservativos, principalmente durante a fase crônica. Lembrar que todas as complicações tardias advêm do processo cicatricial instaurado e todas as medidas clínicas ou cirúrgicas necessárias para a preservação da superfície ocular são importantes ${ }^{(13)}$. Dentre os procedimentos cirúrgicos utilizados em nosso trabalho destacam-se: lise de simbléfaro, rotação marginal, epilação, "patch" corneano, transplante penetrante, transplante de mucosa bucal, transplante de limbo e membrana amniótica. O uso de lente de contato gelatinosa terapêutica ou rígida escleral tem se mostrado uma opção para aqueles casos onde há trauma das margens palpebrais ceratinizadas e cílios aberrantes, proporcionando uma proteção mecânica à frágil superfície corneana ${ }^{(13)}$.

\section{CONCLUSÃO}

Um estudo desta natureza tem suas limitações, pois há a dificuldade na obtenção dos dados por não dispormos dos registros dos pacientes e as informações prestadas pelos mesmos podem estar subestimadas ou errôneas. Mesmo assim, acreditamos que esse estudo seja importante na compreensão dessa afecção em nosso meio, o que pode direcionar melhor a terapêutica e novos estudos mais controlados.

\section{ABSTRACT}

Purpose: To evaluate the epidemiology, possible etiologic factors, complications and treatment of a group of patients with ocular complications secondary to Stevens-Johnson syndrome. Methods: Twenty-two consecutive patients with Stevens-Johnson syndrome were studied. The patients were examined according to the following protocol: identification, previous clinical history, systemic and ophthalmologic manifestations and possible etiologic factors. Results: A total of 22 patients with Stevens-Johnson syndrome were identified. Fifteen patients (68\%) were female, 7 (32\%) male. Ten patients were caucasian (45.4\%), 9 brownish (22\%), 2 black (9\%) and 1 yellow (4.5\%). Mean age was 27.1 (8 to 62). Medications were the most commonly identified etiologic factor (90.9\%), followed by skin herpetic infection (4.5\%) and idiopathic (4.5\%). Dipirone (36.3\%) was the most frequently identified agent, followed by seizure medications $(22.7 \%)$, non-steroidal antiinflammatory drugs $(13.6 \%)$, sulfonamides $(9.0 \%)$, penicillin $(4.5 \%)$, spironolactone $(4.5 \%)$ and dihydroprogestagen and stradiol (4.5\%). Twenty-one patients (95.4\%) had ophthalmologic complications and sixteen patients $(72.7 \%)$ underwent ophthalmologic surgical procedures. Conclusions: The results of this study show important epidemiological aspects of Stevens-Johnson syndrome in our environment, specially related to age, etiology and ocular complications.

Keywords: Stevens-Johnson syndrome/epidemiology; Drug hypersensitivity; Corneal opacity; Visual acuity

\section{REFERÊNCIAS}

1. Stewart MG, Duncan NO $3^{\text {rd }}$, Franklin DJ, Friedman EM, Sulek M. Head and neck manifestations of erythema multiform in children. Otolaryngol Head Neck Surg. 1994;111(3 Pt 1):236-42. 
2. Power WJ, Ghoraishi M, Merayo-Lloves J, Neves RA, Foster CS. Analysis of the acute ophthalmic manifestations of the erythema multiforme/Stevens-Johnson syndrome/toxic epidermal necrolysis disease spectrum. Ophthalmology. 1995; 102(11):1669-76.

3. Manders SM. Serious and life-threatening drug eruptions. Am Fam Physician. 1995;51(8):1865-72.

4. Nishida K, Yamanishi K, Yamada K, Dota A, Kawasaki S, Quantock AJ, et al. Epithelial hyperproliferation and transglutaminase 1 gene expression in Stevens-Johnson syndrome conjunctiva. Am J Pathol. 1999;154(2):331-6.

5. Roujeau JC, Kelly JP, Naldi L, Rzany B, Stern RS, Anderson T, et al. Medication use and the risk of Stevens-Johnson syndrome or toxic epidermal necrolysis. N Engl J Med. 1995;333(24):1600-7.

6. Leenutaphong V, Sivayathorn A, Suthipinittharm P, Sunthonpalin P. StevensJohnson syndrome and toxic epidermal necrolysis in Thailand. Int J. Dermatol. 1993;32(6):428-31.

7. Hodge WG, Discepola MJ, Deschênes J. Adenoviral keratoconjunctivitis precipitating Stevens-Johnson syndrome. Can J Ophthalmol. 1994;29(4):198-200.
8. Kivelä T, Tervo K, Ravila E, Tarkkanen A, Virtanen I, Tervo T. Pseudomembranous and membranous conjunctivitis. Acta Ophthalmol (Copenh). 1992; 70(4):534-42.

9. Assier H, Bastuji-Garin S, Revuz J, Roujeau JC. Erythema multiforme with mucous membrane involvement and Stevens-Johnson syndrome are clinically different disorders with distinct causes. Arch Dermatol. 1995;131(5):539-43. Comment in: Arch Dermatol. 1996;132(6):711-2.

10. Wilkins J, Morrison L, White CR Jr. Oculocutaneous manifestations of the erythema multiform/Stevens-Johnson syndrome/toxic epidermal necrolysis spectrum. Dermatol Clin.. 1992;10(3):571-82.

11. Foster CS, Fong LP, Azar D, Kenyon KR. Episodic conjunctival inflammation after Stevens-Johnson syndrome. Ophthalmology. 1988;95(4):453-62.

12. Bessant DA, Dart JK. Lamellar keratoplasty in the management of inflammatory corneal ulceration and perforation. Eye. 1994;8(Pt 1):22-8.

13. Gomes JAP, Komagome CR, Santos NC, Cunha MC, Freitas D. Membrana amniótica nas cirurgias reconstrutivas da superfície ocular nas ceratoconjuntivites cicatriciais. Arq Bras Oftalmol. 1999;62(5):562-76.

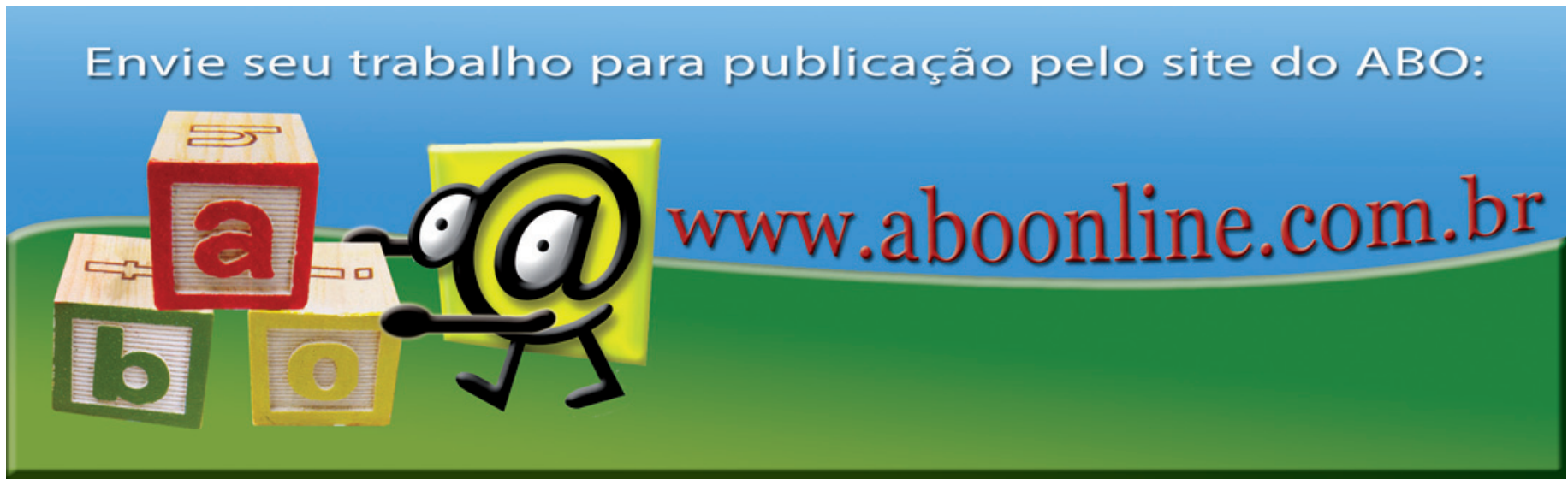

